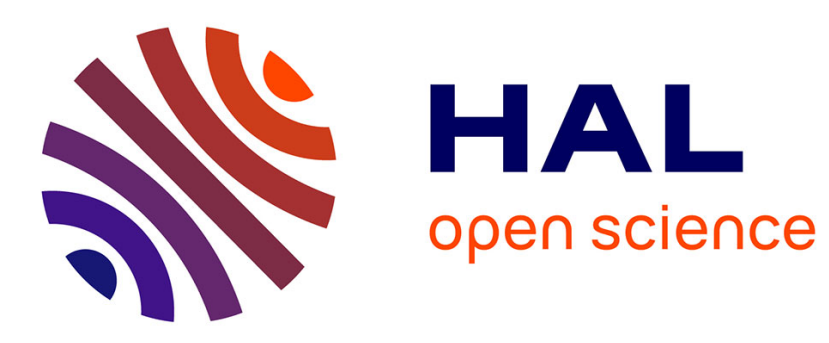

\title{
Batch compressive sensing for passive radar range-Doppler map generation
}

Weike Feng, Jean Friedt, Grigory Cherniak, Motoyuki Sato

\section{To cite this version:}

Weike Feng, Jean Friedt, Grigory Cherniak, Motoyuki Sato. Batch compressive sensing for passive radar range-Doppler map generation. IEEE Transactions on Aerospace and Electronic Systems, 2019, 0, pp.1 - 1. hal-02370729

\section{HAL Id: hal-02370729 \\ https://hal.science/hal-02370729}

Submitted on 19 Nov 2019

HAL is a multi-disciplinary open access archive for the deposit and dissemination of scientific research documents, whether they are published or not. The documents may come from teaching and research institutions in France or abroad, or from public or private research centers.
L'archive ouverte pluridisciplinaire HAL, est destinée au dépôt et à la diffusion de documents scientifiques de niveau recherche, publiés ou non, émanant des établissements d'enseignement et de recherche français ou étrangers, des laboratoires publics ou privés. 


\title{
Batch compressive sensing for passive radar range- Doppler map generation
}

\author{
Weike Feng, Student member, IEEE, Jean-Michel Friedt, Grigory Cherniak, and Motoyuki Sato, \\ Fellow, IEEE
}

\begin{abstract}
By exploiting the sparsity of the scene containing only a few moving targets, a high-resolution and real-time rangeDoppler map generation algorithm for passive bistatic radar is proposed. The proposed algorithm divides the long integration time into multiple short batches, from which a few batches are randomly selected on the basis of compressive sensing theory. A one-dimensional cross-correlation is performed for each selected batch to obtain the range-compressed profile. Mean-value subtraction is then performed to suppress the direct path interference and stationary target reflections. Finally, an extended orthogonal matching pursuit algorithm is proposed for the effective estimation of target Doppler frequency. Practical application of this novel algorithm is examined by the detection of airplanes and ships via two synchronized general-purpose software-defined radio receivers. The results show that the proposed algorithm can achieve an improved resolution and a reduced sidelobe level compared to the conventional algorithms.
\end{abstract}

Index Terms-Passive bistatic radar, range-Doppler map, software-defined radio, batches algorithm, compressive sensing.

\section{INTRODUCTION}

$\mathrm{P}$ ASSIVE bistatic radar (PBR) has attracted considerable attention over the last decades because of its large coverage area, low vulnerability, and reduced electromagnetic pollution to the environment [1], [2]. Different types of illuminators of opportunity (IO), including frequency modulated broadcast emitters [3], digital audio broadcasting emitters [4], spaceborne and Global Navigation Satellite System (GNSS) sources [5], [6], digital communication networks such as Wi-Fi [7], [8], and digital terrestrial television broadcasting (DTTB) emitters [9], [10], have been exploited in PBR to detect and track air and coastal targets. Various signal processing techniques transposed from active radar to passive radar such as space-time adaptive processing (STAP) [11], [12], synthetic aperture radar (SAR) imaging [9], [13], and inverse SAR (ISAR) imaging [14], [15] have been developed for specific applications.

In a PBR system, the bistatic range and Doppler frequency of the moving target are estimated by calculating the twodimensional (2D) cross ambiguity function (CAF). Typically, two approaches, namely, the Fourier transform method and the

This work was supported by JSPS Grant-in-Aid for Scientific Research (A) 26249058. The DVB-T passive radar investigation is partly supported by the French Centre National de la Recherche Scientifique (CNRS) PEPS grant.

Weike Feng and Grigory Cherniak are with the Graduate School of Environmental Studies, Tohoku University, 980-8579, Sendai, Japan (e-mail: feng.weike.q4@dc.tohoku.ac.jp and grigory.chernyak.s6@dc.tohoku.ac.jp) cross-correlation method [2], are used to generate target rangeDoppler maps by implementing the fast Fourier transform (FFT). However, for long-term measurements with a high data sampling frequency, the computational burden of these approaches is usually unacceptable, especially when sufficient signal processing gain and fine range-Doppler resolution are desired for a large surveillance area. Therefore, some suboptimal algorithms [16], [17] have been proposed to obtain real-time processing capability. In particular, the batches algorithm, which is analogous to pulse-Doppler processing in classical active pulse-Doppler radar, has been proposed and validated in [18]. In this algorithm, a long received signal is divided into many short batches. Then, range compression is conducted for each batch by a one-dimensional (1D) crosscorrelation without compensating for the target Doppler shift. Finally, the Fourier transform along each range bin is conducted to estimate the Doppler frequency of the target. The computing time required to generate the target range-Doppler map by the batches algorithm is considerably less than that required by lossless approaches.

When the matched filter theory is used for Doppler estimation, the range-Doppler maps obtained in a limited integration time have a limited resolution and high sidelobe level. The sidelobes produced by the strong targets introduce spurious values in their neighboring Doppler cells, thus leading to wrong position and amplitude estimations of the weak targets. Moreover, FFT will calculate Doppler frequencies up to half of the inverse of the batch duration. When a shorter batch length is used to achieve a lower signal-to-noise ratio (SNR) loss, the maximal calculated Doppler frequency is considerably larger than the Doppler frequency of the target. Therefore, many calculated values of FFT are meaningless and hence ignored. Although a simple way to overcome this is to use the decimation technique before the Fourier transform, this leads to additional computational complexity and SNR loss. Recently, compressive sensing (CS) theory [19-21] has been extensively studied to overcome the sidelobe and resolution problems commonly encountered in the field of radar signal processing, such as ISAR [22-24], SAR [25-27], STAP [28], [29], and passive radar range-Doppler map generation [30], [31]. In

\footnotetext{
Jean-Michel Friedt is with the FEMTO-ST, Time \& Frequency department, Besancon, France. (e-mail: jean-michel.friedt@femto-st.fr).

Motoyuki Sato is with the Center for Northeast Asian Studies, Tohoku University, 980-8576, Sendai, Japan (e-mail: motoyuki.sato.b3@tohoku.ac.jp). Corresponding author: Weike Feng.
} 
comparison with conventional approaches, CS theory can achieve higher resolution and better performance. Furthermore, based on the signal sparsity in a specific dictionary and by solving a restricted minimization problem, CS theory can recover the sparse signal from its undersampled measurement with a high probability. Undersampling can reduce the computational complexity and memory usage, making CS theory attractive for practical applications.

In this study, we propose a novel algorithm based on CS to reduce the sidelobe level obtained when using the batches algorithm for CAF calculation in PBR by exploiting the sparsity of the scene. In addition, this algorithm allows processing within a specific Doppler frequency range. For typical applications of PBR, the moving targets are always sparse in the range-Doppler domain after removing direct path interference (DPI) and strong stationary target reflections (such as islands in coastal applications). However, if CS is directly applied to PBR with a long integration time and high sampling frequency, as studied by the previous researchers [32], [33], the computational complexity may prevent real-time application. Therefore, based on the principle of the batches algorithm, the long integration time is divided into multiple short batches, from which only a few batches are randomly selected and range compression is correspondingly conducted. Finally, we propose an extended orthogonal matching pursuit (EOMP) algorithm to effectively estimate the Doppler frequency of the targets. Compared to the batches algorithm, the proposed algorithm can achieve range-Doppler maps with an improved resolution and a reduced sidelobe level while maintaining the real-time processing capacity. These concepts were experimentally demonstrated using a low-cost PBR system implemented with digital video broadcasting-terrestrial (DVB-T) receivers, as described in detail in the accompanying article describing the hardware setup [34].

\section{SIGNAL MODEL}

In a PBR system, the reference signal received by an antenna directly oriented to IOs (e.g., TV towers) can be expressed as

$$
s_{\text {ref }}(t)=A_{\text {ref }} s_{0}\left(t-t_{\text {ref }}\right)+n_{\text {ref }}(t)
$$

where $A_{\text {ref }}$ is the complex amplitude, $s_{0}(t)$ is the transmitted signal, $t_{\text {ref }}$ denotes the transmission time from the IOs to the reference antenna, and $n_{\text {ref }}(t)$ denotes the additive noise.

On the other hand, the received signal from a surveillance antenna, which includes DPI, multipath echoes, and moving target reflections, is given by

$$
\begin{aligned}
s_{\text {surv }}(t) & =A_{\text {surv }} s_{0}\left(t-t_{\text {surv }}\right)+\sum_{m=1}^{M} a_{m} s_{0}\left(t-t_{m}\right) \\
& +\sum_{p=1}^{P} a_{p} s_{0}\left(t-t_{p}\right) e^{j 2 \pi f_{p} t}+n_{\text {surv }}(t)
\end{aligned}
$$

where $A_{\text {surv }}$ is the amplitude of the DPI signal with time delay $t_{\text {surv }}$, which is equal to $t_{\text {ref }}$ when the reference and surveillance antennae are co-located, and $M, a_{m}$, and $t_{m}$ are the number, amplitude, and time delay of the multipath echoes, respectively. The third term on the right side of (2) denotes the moving target components, where $f_{p}$ is the Doppler frequency of the $p$-th moving target; $P, a_{p}$, and $t_{p}$ are the number, amplitude, and time delay of the moving targets; and $n_{\text {surv }}(t)$ denotes the additive noise in the surveillance channel.

The classical way to estimate the bistatic range and Doppler frequency of the moving targets is to calculate the CAF between the received reference and surveillance signals. The CAF can be expressed as

$$
\chi(\tau, f)=\int_{0}^{T_{i t t}} s_{\text {surv }}(t) s_{\text {ref }}^{*}(t-\tau) e^{-j 2 \pi f t} d t
$$

where $\chi(\tau, f)$ represents the range-Doppler map of the targets, $\tau$ and $f$ are the expected bistatic time delay and Doppler shift of the reflected signal from the target, respectively, $(\cdot)^{*}$ denotes the complex conjugate, and $T_{\text {int }}$ denotes the integration time that determines the system Doppler resolution. Notably, to avoid integration losses in (3) [18], the integration time is chosen as $T_{\text {int }}=T_{\text {ref }}+\tau_{\max }$, where $T_{\text {ref }}$ is the length of the reference signal and $\tau_{\max }$ is the maximal time delay to be processed. In this case, it is assumed that $s_{r e f}(t)=0$ when $t \in\left(T_{r e f}, T_{\text {int }}\right]$.

The most straightforward approach to obtain the rangeDoppler map of the target is to calculate (3) for each pair of bistatic time delay in $\left(\tau_{\min }, \tau_{\max }\right)$ and Doppler frequency in $\left(f_{\min }\right.$, $f_{\max }$ ). However, the computational complexity is normally beyond that manageable in real-time processing, especially for large surveillance areas and fast-moving targets which have large Doppler frequencies. Two simpler methods, namely, the Fourier transform method and the cross-correlation method, can be used. In the first method, the Fourier transform of $s_{\text {surv }}(t) \cdot s^{*}$ ref $(t-\tau)$ for a given time delay $\tau$ is obtained using FFT. In the second method, the cross-correlation between the Doppler-shifted reference signal for a given Doppler frequency $f$, i.e., $S_{r e f}(t) e^{-\mathrm{j} 2 \pi f t}$, and the surveillance signal $s_{\text {surv }}(t)$ is calculated by FFT.

Although these two approaches can reduce the computational cost, two drawbacks limit their applications [18]. First, FFT generates some unused Doppler frequency values in the first method or unused bistatic range values in the second method. Second, if high signal processing gain and range-Doppler resolution are desired, the long integration time and high data sampling frequency may make the calculation of (3) via FFT impractical. To solve these problems, several suboptimal algorithms have been proposed. In particular, for the first problem, based on the prior knowledge of target distance and velocity, the decimation technique is applied before the Fourier transform process. For the second problem, the batches algorithm is proposed, which divides the long integration time into multiple short batches, and this method will be reviewed in the next section.

\section{BATCH COMPRESSIVE SENSING ALGORITHM}

\section{A. Batches algorithm review}

In this subsection, the batches algorithm is briefly reviewed to provide the basis for the proposed algorithm. In the batches algorithm, a dataset collected over a long time is divided into multiple short batches. According to [14], the $n$-th $(n=1,2, \ldots, N)$ batch of the reference signal can be expressed as

$$
S_{\text {ref }}(t, n)=S_{\text {ref }}\left(t+n T_{b}\right) \operatorname{rect}\left[\left(t-T_{b} / 2\right) / T_{b}\right]
$$

where $T_{b}$ is the length of each batch and is assumed to be given by $T_{b}=T_{\text {ref }} / N$ without loss of generality and $\operatorname{rect}(x)$ is unity 
when $-1 / 2 \leq x \leq 1 / 2$ and is zero otherwise.

For the surveillance signal, to guarantee the maximum integration gain in each batch, a partially overlapping division approach is employed and the $n$-th $(n=1,2, \ldots, N)$ batch is expressed as

$$
S_{\text {surv }}(t, n)=S_{\text {surv }}\left(t+n T_{b}\right) \operatorname{rect}\left(\frac{t-\left(T_{b}+\tau_{\max }\right) / 2}{T_{b}+\tau_{\max }}\right)
$$

Thus, the reference and surveillance signals can be rewritten as

$$
\left\{\begin{array}{l}
S_{\text {ref }}(t)=\sum_{n=1}^{N} S_{\text {ref }}\left(t-n T_{b}, n\right) \\
S_{\text {surv }}(t)=\sum_{n=1}^{N} S_{\text {surv }}\left(t-n T_{b}, n\right)
\end{array}\right.
$$

Then, by substituting (6) into (3), we get

$$
\begin{aligned}
& \chi(\tau, f) \\
& =\sum_{n=1}^{N} e^{-j 2 \pi f(n-1) T_{b}} \int_{0}^{T_{b}+\tau_{\max }} S_{\text {surv }}(t, n) S_{r e f}^{*}(t-\tau, n) e^{-j 2 \pi f t} d t
\end{aligned}
$$

Defining the slow time as $u=\left[0, T_{b}, \ldots,(N-1) T_{b}\right]$ and the $n$ th ambiguity function as

$$
\chi_{n}(\tau, f)=\int_{0}^{T_{b}+\tau_{\max }} S_{\text {surv }}(t, n) S_{r e f}^{*}(t-\tau, n) e^{-j 2 \pi f t} d t
$$

(7) can be represented as

$$
\chi(\tau, f)=\sum_{n=1}^{N} e^{-j 2 \pi f u_{n}} \chi_{n}(\tau, f)
$$

From (9), the optimal CAF can be estimated as a weighted sum of the ambiguity functions of all batches. Moreover, if the product of the batch length and the maximum target Doppler frequency is small, then the phase of each sampling in the batch is closer to the phase of the sampling of the central time, i.e., $2 \pi f t \approx \pi f T_{b}, \forall t \in\left[0, T_{b}\right] \quad$ and $e^{-j 2 \pi f t} \approx e^{-j \pi f T_{b}}, \forall t \in\left[0, T_{b}\right]$. Thus, (8) can be approximated as

$$
\chi_{n}(\tau, f) \approx e^{-j \pi f T_{b}} \int_{0}^{T_{b}+\tau_{\max }} S_{\text {surv }}(t, n) S_{r e f}^{*}(t-\tau, n) d t
$$

Defining the cross-correlation within the $n$-th batch as

$$
\chi_{n}(\tau)=\int_{0}^{T_{b}+\tau_{\max }} S_{\text {surv }}(t, n) S_{r e f}^{*}(t-\tau, n) d t
$$

(9) can be approximated as

$$
\chi(\tau, f) \approx e^{-j \pi f T_{b}} \sum_{n=1}^{N} e^{-j 2 \pi f u_{n}} \chi_{n}(\tau)
$$

Based on (12), the range-Doppler map of the target can be effectively derived using FFT. Specifically, the 1D crosscorrelation can be applied to each batch to obtain the rangecompressed profiles and then the Fourier transform along the slow times can be conducted to estimate the Doppler frequencies of the targets. It should be mentioned that after obtaining the Fourier transform for each range bin, $\mathrm{e}^{-\mathrm{j} \pi f T b}$ should be multiplied accordingly. However, if the target Doppler shift can be ignored for each batch, i.e., $e^{-j 2 \pi f t} \approx 1$ for $\forall t \in\left[0, T_{b}\right]$, the first exponential function in (12) can be ignored. We have observed that the approximations $\mathrm{e}^{-\mathrm{j} 2 \pi f t} \approx \mathrm{e}^{-\mathrm{j} \pi f T h}$ and $\mathrm{e}^{-\mathrm{j} 2 \pi f \mathrm{t}} \approx 1$ do not show much difference experimentally.

To achieve acceptable approximation precision, the batch length should be short enough to reduce the loss of SNR caused by the batch-wise approximation [14] compared with the optimal process, especially for a fast target with high Doppler frequency. However, the computational complexity of the batches algorithm increases with decreasing batch length (i.e., the number of batches increases). Therefore, a suitable batch length (and hence number of batches) should be considered to ensure a good trade-off between accuracy and computational complexity.

\section{B. Range-Doppler map generation with CS model}

Because the matched filter theory is used, conventional algorithms, including the batch algorithm, have a limited rangeDoppler resolution owing to the limited frequency bandwidth and integration time. In addition, the Fourier transform-based approach of these algorithms produces high sidelobe levels. The sidelobes of strong targets mask the weaker targets, making processes such as detection and tracking difficult. In general, amplitude weighting methods can be used to reduce the sidelobe level, however, these methods affect the resolution.

CS theory can also be easily applied for PBR [32], [33]. First, expected time delays in $\left(\tau_{\min }, \tau_{\max }\right)$ and Doppler frequencies in $\left(f_{\min }, f_{\max }\right)$ are discretized into $I$ and $J$ grids. Then, a sparsifying dictionary $\boldsymbol{\Psi}$ can be designed with the $((j-1) I+i)$-th column calculated by the reference signal with time delay $\tau_{i}$ and Doppler frequency $f_{j}$, i.e., $S_{r e f}\left(t-\tau_{i}\right) e^{-\mathrm{j} 2 \pi f j t}$. Correspondingly, the range-Doppler estimation problem is modeled as

$$
\boldsymbol{s}_{\text {surv }}=\Psi \chi+\boldsymbol{n}
$$

where $\boldsymbol{s}_{\text {surv }}$ is the vectorized surveillance signal obtained by stacking $s_{\text {surv }}(t)$ with all samples, $\chi$ is the vectorized rangeDoppler map, and $\boldsymbol{n}$ is the noise vector. To reduce the complexity and memory used, a random selection approach can be applied, giving

$$
\boldsymbol{s}_{\text {surv }}^{u n}=\boldsymbol{\Phi} \boldsymbol{S}_{\text {surv }}=\boldsymbol{\Phi} \boldsymbol{\Psi} \chi+\boldsymbol{\Phi} \boldsymbol{n}=\Psi^{u n} \chi+\boldsymbol{n}^{u n}
$$

where the superscript "un" stands for "undersampling", $\Phi$ is the measure matrix used to randomly select rows from $\boldsymbol{\Psi}$, and the number of rows indicate the selected samplings from $\boldsymbol{s}_{\text {surv }}$.

Then, based on CS theory and assuming that the rangeDoppler vector is sparse, i.e., there are only a few targets in the surveillance area, the solution of (14) is given by solving the following optimization problem:

$$
\boldsymbol{X}=\min \|\boldsymbol{X}\|_{0}, \text { s.t. }\left\|\boldsymbol{s}_{\text {surv }}^{u n}-\boldsymbol{\Psi}^{u n} \boldsymbol{\chi}\right\|_{2}^{2} \leq \varepsilon
$$

where $\varepsilon$ is the noise level.

Although it has been shown to have non-deterministic polynomial-time hardness, (15) can be approximately solved by many state-of-the-art algorithms, such as OMP algorithm [35], smoothed $l_{0}$ norm algorithm [36], and basis pursuit denoising algorithm [37]. However, two problems may make (15) unsuitable for practical application. The first is the considerable computational and memory requirement. Long computing time makes it impossible for real-time application, especially when high sampling rate, long integration time, fine range-Doppler resolution, and large range-Doppler coverage are desired. The second problem, caused by the insufficient sparsity assumption of the range-Doppler map, may occur when DPI is strong and multiple stationary targets are included in the scene. In such a case, the sparsity of the target range-Doppler map is destroyed. Although some algorithms, such as the normalized least mean squares algorithm [38], [39], can be employed to suppress DPI and multipath echoes, higher computational complexity is inevitable. In this subsection, we propose a novel CS-based 
range-Doppler estimation model for PBR to solve these two problems.

First, similar to the process in the batch algorithm, the longtime received signal is divided into multiple short batches. Then, following the Doppler frequency approximation, 1D crosscorrelation is applied to each batch using FFT, which gives us

$$
\begin{aligned}
\chi_{n}(\tau) & =\int_{0}^{T_{b}+\tau_{\max }} S_{\text {surv }}(t, n) S_{\text {ref }}^{*}(t-\tau, n) d t \\
& =\chi_{n}^{0}(\tau)+\sum_{m=1}^{M} \chi_{n}^{m}(\tau)+\sum_{p=1}^{P} \chi_{n}^{p}(\tau)
\end{aligned}
$$

where $\chi_{n}^{0}(\tau), \chi_{n}^{m}(\tau)$, and $\chi_{n}^{p}(\tau)$ are the DPI component, $m$ th multipath component, and $p$-th moving target component in the $n$-th range profile, respectively. Because the range profiles of DPI and stationary targets show small changes in different batches, the mean value subtraction process is employed to suppress their influence, which is given by

$$
\chi_{n}^{\text {tar }}(\tau)=\chi_{n}(\tau)-\sum_{n=1}^{N} \chi_{n}(\tau) / N
$$

where the superscript "tar" represents "targets."

Therefore, we have a signal matrix consisting of range profiles of all batches, which is defined as

$$
\boldsymbol{S}=\left[\begin{array}{ccc}
\chi_{1}^{\text {tar }}\left(\tau_{\min }\right) & \cdots & \chi_{N}^{\text {tar }}\left(\tau_{\min }\right) \\
\vdots & \ddots & \vdots \\
\chi_{1}^{\text {tar }}\left(\tau_{\max }\right) & \cdots & \chi_{N}^{\text {tar }}\left(\tau_{\max }\right)
\end{array}\right] \in C^{N_{\text {bin }} \times N}
$$

where $N_{b i n}$ is the number of range bins.

Then, based on (12), the batches algorithm can be reformulated to get the target range-Doppler map as $\boldsymbol{X}=\boldsymbol{S} \boldsymbol{\Psi}_{D} \in C^{N_{\text {bin }} \times N_{\text {Dop }}}$, where $\boldsymbol{\Psi}_{D}$ is the Doppler-shifted Fourier transform matrix and is expressed as

$$
\Psi_{D}=\left[\begin{array}{ccc}
e^{-j \pi f_{\min } T_{b}} e^{-j 2 \pi f_{\min } u_{1}} & \cdots & e^{-j \pi f_{\max } T_{b}} e^{-j 2 \pi f_{\max } u_{1}} \\
\vdots & \ddots & \vdots \\
e^{-j \pi f_{\min } T_{b}} e^{-j 2 \pi f_{\min } u_{N}} & \cdots & e^{-j \pi f_{\max } T_{b}} e^{-j 2 \pi f_{\max } u_{N}}
\end{array}\right] \in C^{N \times N_{D o p}}
$$

where $N_{D o p}$ is the number of Doppler frequencies.

Using the transpose process, we obtain $\boldsymbol{X}^{\mathrm{T}}=\left(\boldsymbol{\Psi}_{D}\right)^{\mathrm{T}} \boldsymbol{S}^{\mathrm{T}}$, which can be considered as a solution obtained by the Fourier transform of the following problem:

$$
\boldsymbol{S}^{\mathrm{T}}=\left(\boldsymbol{\Psi}_{D}\right)^{*} \boldsymbol{X}^{\mathrm{T}}
$$

where $(\cdot)^{\mathrm{T}}$ denotes the transpose.

However, as mentioned above, because of the limited integration time, the Fourier transform-based solution of (20) has a limited resolution and high sidelobe level. For most applications, after DPI and multipath echo suppression, the range-Doppler map is always sparse, i.e., only few elements corresponding to the moving targets in $\boldsymbol{X}$ have significant values. Therefore, if the sparse property of $\boldsymbol{X}$ can be exploited, better resolution and a lower sidelobe level can be obtained. Therefore, based on CS theory, we propose to solve the following restricted optimization problem to estimate the rangeDoppler parameters of the targets:

$$
\boldsymbol{X}=\min \|\boldsymbol{X}\|_{0}, \text { s.t. }\left\|\boldsymbol{S}_{0}-\boldsymbol{\Psi}_{D}^{0} \boldsymbol{X}^{\mathrm{T}}\right\|_{F}^{2} \leq \varepsilon
$$

where $\boldsymbol{S}_{0}=\boldsymbol{S}^{\mathrm{T}}, \boldsymbol{\Psi}_{D}^{0}=\left(\boldsymbol{\Psi}_{D}\right)^{*}$, and $\|\cdot\|_{F}$ denote the Frobenius norm of a matrix. To further exploit the advantage of CS theory to reduce computational complexity, the batches are randomly selected, resulting in

$$
\boldsymbol{X}=\min \|\boldsymbol{X}\|_{0} \text {, s.t. }\left\|\boldsymbol{S}_{1}-\boldsymbol{\Psi}_{1} \boldsymbol{X}^{\mathrm{T}}\right\|_{F}^{2} \leq \varepsilon
$$

where $\boldsymbol{S}_{1}=\boldsymbol{\Phi}_{1} \boldsymbol{S}_{0} \in C^{N_{s} \times N_{\text {bin }}}$ is the undersampled signal matrix, $\boldsymbol{\Psi}_{1}=\boldsymbol{\Phi}_{1} \boldsymbol{\Psi}_{D}^{0} \in C^{N_{s} \times N_{D o p}}$ denotes the defined sparsifying matrix, $\Phi_{1} \in C^{N_{s} \times N}$ is the measure matrix used to randomly select batches, and $N_{s}$ is the number of selected batches. Notably, to save memory usage and reduce computational complexity, undersampling should be performed just after the determination of batches. In such a case, the signal processes mentioned above should be adjusted accordingly.

\section{Extended OMP algorithm}

In this subsection, we attempt to solve (22) effectively and accurately. Although the $l_{0}$ norm in (22) is the best to measure the sparsity of a signal in the absence of noise, its computational burden is very high because of its discontinuity. Therefore, some advanced algorithms should be adopted to solve (22). Compared to (15), the difference in the proposed model is that it is established in the matrix form. For the classical 1D CS problem in (15), three different algorithm types, i.e., greedy algorithms [40], [41], Bayesian CS algorithms [42], and convex optimization algorithms [43], have been extensively studied and applied. In general, greedy algorithms are much more computationally efficient than other algorithms and are easier to implement practically. Moreover, among greedy algorithms, the OMP algorithm [35] is widely used because of its simplicity and efficiency. Therefore, we extend the OMP algorithm, which is normally used to solve sparse vector recovery problems, to the sparse matrix recovery problem in (22). The basic idea of the OMP algorithm is to sequentially find the support set of the measured signal and then project it on the atoms selected from the sparsifying dictionary. Following this basic idea, we propose an extended OMP (EOMP) algorithm, as summarized in Table I.

TABLE I. EOMP ALGORITHM

Input: $\boldsymbol{S}_{1}, \boldsymbol{\Psi}_{1}$, and iteration number $Q$.

Procedure:

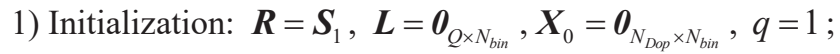

2) Index finding: $\left[a_{q}, b_{q}\right] \leftarrow \arg \max \left|\Psi_{1}^{\mathrm{H}} \boldsymbol{R}\right|$, where $a_{q}$ and $b_{q}$ are the row index and column index of the biggest element in $\left|\Psi_{1}^{\mathrm{H}} \boldsymbol{R}\right|$, and $(\cdot)^{\mathrm{H}}$ denotes the conjugate transpose;

3) Index set expansion: $\boldsymbol{L}\left(q, b_{q}\right)=a_{q}$, and $\boldsymbol{K}=\operatorname{Ind}\left\{\boldsymbol{L}\left[b_{q}\right]\right\}$, where $\operatorname{Ind}\{\cdot\}$ denotes the nonzero elements in a vector and $Z[u]$ denotes the $u$-th column of matrix $\boldsymbol{Z}$;

4) Estimation: $\Theta=\Psi_{1}[\boldsymbol{K}], \boldsymbol{x}=\Theta^{\dagger} \boldsymbol{S}_{1}\left[b_{q}\right]$, and $\boldsymbol{X}_{0}\left[\boldsymbol{K}, b_{q}\right]=\boldsymbol{x}$, where $(\cdot)^{\dagger}$ is the pseudo-inverse of a matrix;

5) Residual updating: $\boldsymbol{R}\left[b_{q}\right]=\boldsymbol{S}_{1}\left[b_{q}\right]-\boldsymbol{\Theta} \boldsymbol{x}$;

6) Iteration: $q=q+1$, if $q \leq Q$ go back to 2), otherwise, stop. Output: $\boldsymbol{X}=\boldsymbol{X}_{0}^{\mathrm{T}}$.

In each iteration, the atom in $\boldsymbol{\Psi}_{1}$ that is the most strongly correlated with the residual matrix $\boldsymbol{R}$ (the remaining part of $\boldsymbol{S}_{1}$ ) 
is chosen, and the corresponding row index and column index are recorded in $\boldsymbol{L}$. Then, for the signal column selected in this iteration, the contribution of all selected atoms is subtracted by projecting this signal column onto the orthogonal subspace spanned by these atoms. The iteration is stopped when the number of iterations equals the maximum iteration number $Q$. The final estimation of $\boldsymbol{X}$ has non-zero entries corresponding to the index set $\boldsymbol{L}$, and the values of these entries are obtained by applying the pseudo-inversion process. For the practical implementation for PBR range-Doppler mapping problem, we assume that there are $J$ moving targets with different rangeDoppler parameters. In the first iteration, the range and Doppler frequency of the strongest target are estimated by the least squares method. Then, the contribution of this target to the received signal is subtracted. In the next iteration, the secondstrongest target is considered. Finally, all targets are expected to be recovered after $Q \geq J$ iterations.

\section{Signal processing chain and complexity analysis}

In summary, the main steps of the proposed range-Doppler map generation algorithm are shown in Fig. 1 and can be described as follows:

1) With the same approach as that shown in [18], divide the reference signal into $N$ consecutive batches and divide the surveillance signal into $N$ partially overlapping batches.

2) Randomly select $N_{s}$ corresponding batches from the reference and surveillance channels.

3) For each selected batch pair, apply 1D cross-correlation using FFT.

4) Use the mean-value subtraction approach to suppress DPI and stationary target reflections.

5) Given the pre-defined sparsifying dictionary and maximum iteration number, use the proposed EOMP algorithm to generate the range-Doppler map of the targets.

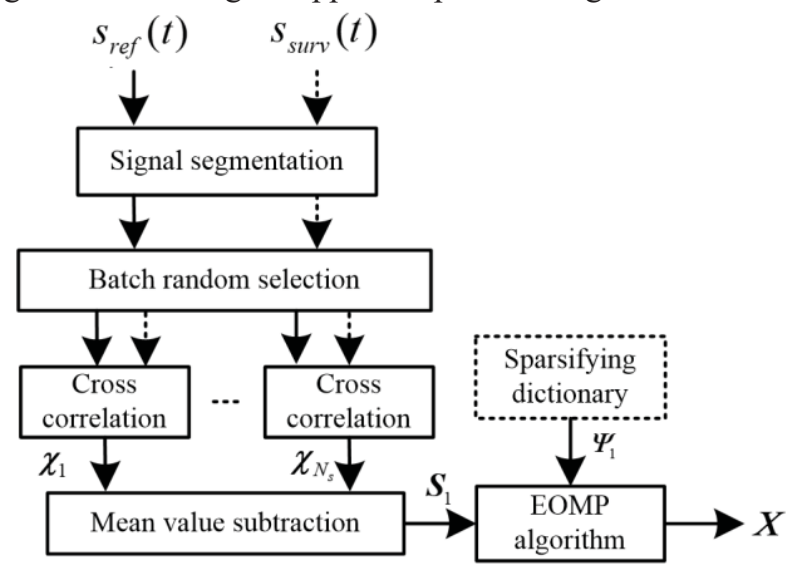

Fig. 1. Flow chart of the proposed range-Doppler map generation algorithm.

Since the real-time processing capacity is considered to be important, the computational complexity of the proposed algorithm is analyzed. The time-consuming parts of the proposed algorithm are the cross-correlation step (item 3 in the algorithm description above) and the EOMP step (item 5). Since FFT can be used for the efficient implementation of the cross-correlation step, the computational burden in terms of complex multiplications for all the selected batches is given by
$3 N_{b i n} N_{s} \log _{2}\left(N_{b i n}\right)$. The index finding step dominates the computational consumption of the EOMP algorithm and the complexities of other steps of this algorithm are negligible. For $Q$ iterations, the total complex multiplication number for index finding is given by $Q N_{b i n} N_{s} N_{\text {Dop }}$. Therefore, the complexity of the proposed algorithm can be approximated as $\left[3 \log _{2}\left(N_{b i n}\right)+Q N_{\text {Dop }}\right] N_{b i n} N_{s}$. Thus, with an increase in the selected batch number, range bin number, iteration number of EOMP, and Doppler frequency number, the computational load increases. However, thanks to the batch segmentation, undersampling, and the proposed efficient greedy algorithm, real-time range-Doppler generation can be obtained on a general-purpose personal computer (PC), similar to the classical batches algorithm.

\section{EXPERIMENTAL RESULTS}

The experimental setup used to collect data is described in [34]: it mainly consists of two clock-synchronized DVB-T receivers used as general-purpose software-defined radio sources. The sampling rate is $2.048 \mathrm{Msamples} / \mathrm{s}$ and data are collected using the GNURadio framework [44] using two Osmocom sources to display real-time cross-correlation peak values for assessing the quality of the data. As the setup is fully powered by a universal serial bus, it can be easily transported, along with the antennas, for on-field measurements. The experiment setups for landing airplanes and multiple ships are shown in Fig. 2.
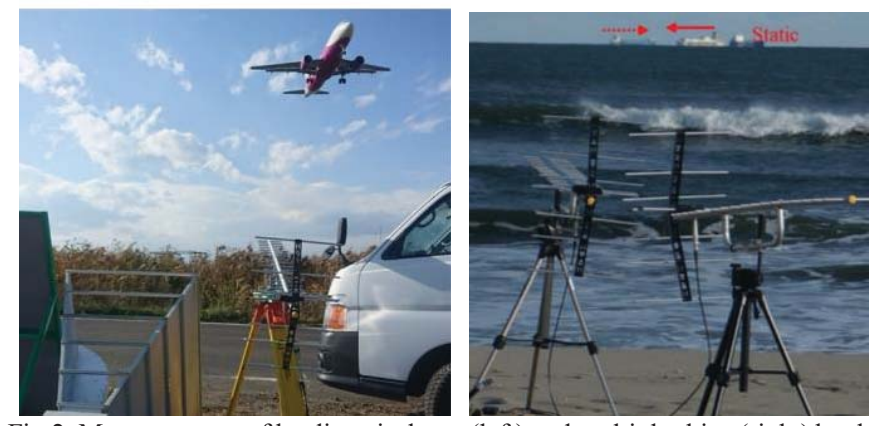

Fig.2. Measurements of landing airplanes (left) and multiple ships (right) by the designed PBR system [34].

\section{A. Fast moving target: landing airplanes}

Landing airplanes are selected as the initial target because of their large cross-section and because their large radial velocity $v$ during landing yields Doppler shifts of the order of hundred hertz. Indeed, considering that the reference signal carrier frequency is approximately $500 \mathrm{MHz}$, the Doppler shift $f$ induced in the carrier frequency $f_{c}$ is given as $f=2 f_{c} v / c$, where $c$ is the speed of light. When $v=100 \mathrm{~m} / \mathrm{s}, f \approx 330 \mathrm{~Hz}$. This value is the upper bound of the expected Doppler shift introduced by the motion of the airplane in a bistatic configuration assuming that the bistatic angle is null. This value also suggests the integration duration and targeted Doppler coverage.

At the beginning, we show the results obtained by the classical batches algorithm. Fig. 3 (a) shows the crosscorrelation results of different batches for a total of $2^{10}$ batches. Fig. 3 (b) presents the mean-value subtracted cross-correlation 
result, with the airplane clearly observed. Fig. 3 indicates that the target can be easily distinguished only after removing DPI and strong stationary target echoes.

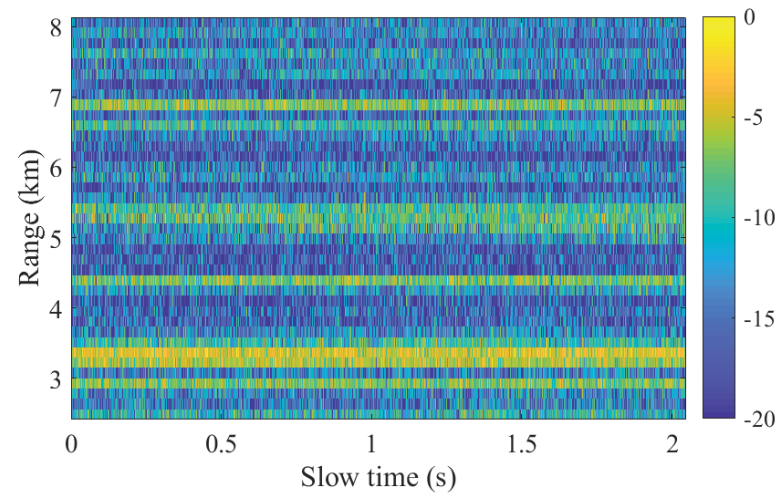

(a) Cross-correlation results

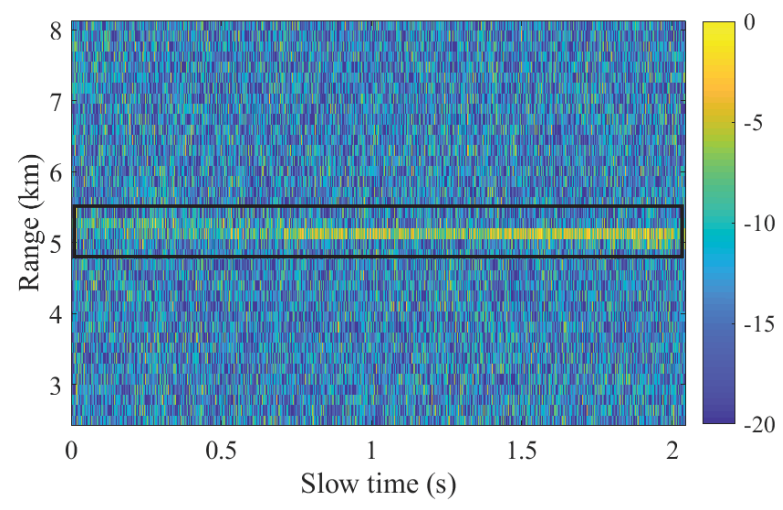

(b) Background subtraction

Fig. 3. (a) Cross-correlation results of different batches, and (b) mean values along the slow time is subtracted from all batches, removing static clutter and leaving only the moving target indicated by the black rectangle.

Therefore, as shown in Fig. 4 (a), the range-Doppler map can be generated by the Fourier transform in the slow time direction. Furthermore, the range-Doppler map considering $2^{14}$ batches with the same steps of range compression and background subtraction is presented in Fig. 4 (b). By shortening the batch length, a higher SNR can be achieved, which can be demonstrated by the integrated sidelobe level (ISL) of the batch algorithm as a function of batch number, as given in Fig. 5. The ISL in $\mathrm{dB}$ scale is defined as

$$
\mathrm{ISL}=10 \log \left(\frac{\sum_{(i, j) \notin \Omega} \chi^{2}\left(\tau_{i}, f_{j}\right)}{\sum_{(i, j) \in \Omega} \chi^{2}\left(\tau_{i}, f_{j}\right)}\right)
$$

where $\Omega$ is defined as a 3-cell-long and 3-cell-wide window around the target.

For short batches, the Doppler shift along the batch length can be ignored. Therefore, as observed in Fig. 5, the sidelobe level is lowered with increasing number of batches, which is consistent with the theoretical analysis. However, the increase in the number of batches induces an increase in the computation time. Furthermore, as shown in Fig. 5, the SNR level is almost constant when the number of batches is greater than $2^{14}$. Therefore, for the proposed algorithm, $2^{14}$ is selected as the base value.

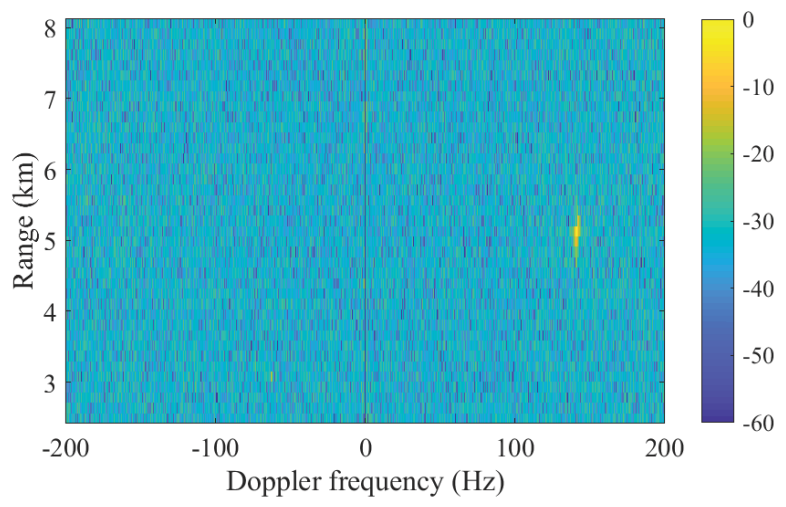

(a) $2^{10}$ batches

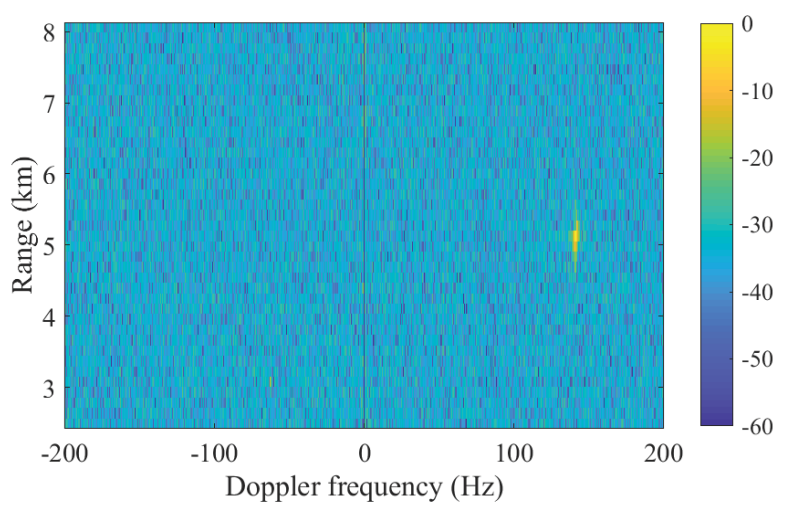

(b) $2^{14}$ batches

Fig. 4. Range-Doppler map resulting from the Fourier transform along the slow time, providing estimation of the Doppler frequencies introduced by target motion. (a) $2^{10}$ batches and (b) $2^{14}$ batches.

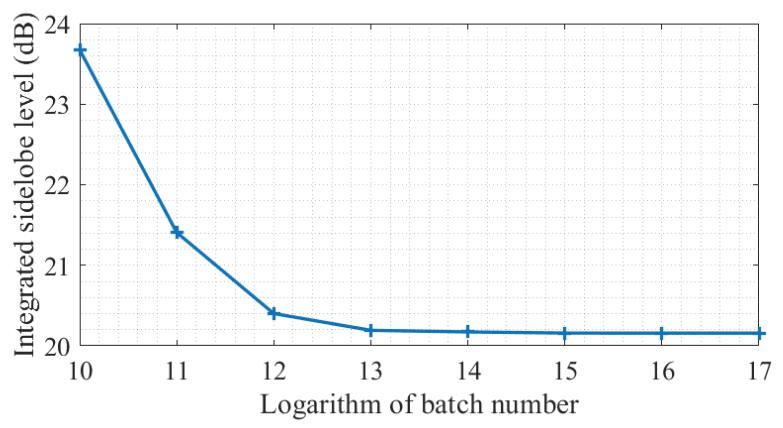

Fig. 5. Sidelobe level of the batches algorithm as a function of the number of batches.

Fig. 6 shows the range-Doppler map obtained by the proposed algorithm with $2^{11}$ batches randomly selected from the $2^{14}$ batches. The iteration number of the proposed EOMP algorithm is set to 10 , which is sufficient to estimate the target. Fig. 6 shows that the proposed algorithm can provide a clearer range-Doppler map with only one outlier (caused by the noise), as indicated by the white square. To show the stability of the proposed algorithm with randomly selected undersampled data, 100 random selections of the batches are conducted and the average value is presented. It is experimentally proved that, with 100 trials, the performance (e.g., the ISL) of the proposed algorithm is statistically converged, as shown in Fig. 7 (a). 
Taking 100 trials will induce many more outliers than the single trial case, as can be seen from Fig. 7 (b). However, for 100 trials, the target can always be recovered, which demonstrates that no matter how random the selected batches are, the proposed algorithm is robust enough to work well. We note that, in practice, only one-time random selection will be conducted to achieve the real-time processing capacity.

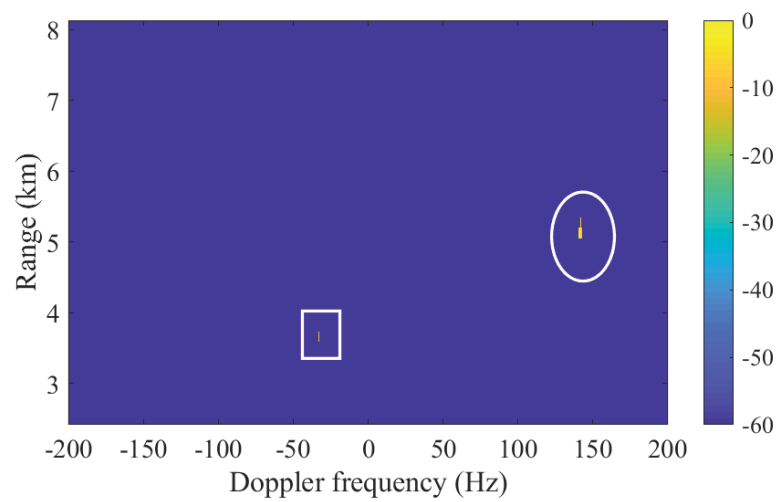

Fig. 6. Proposed algorithm combining the batches algorithm with compressive sensing run on $2^{11}$ randomly selected batches (the white circle indicates target and the white square indicates the outlier).

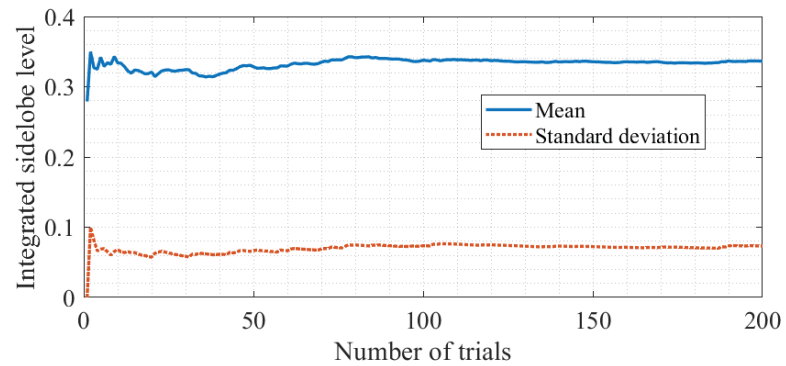

(a) ISL against different number of trials

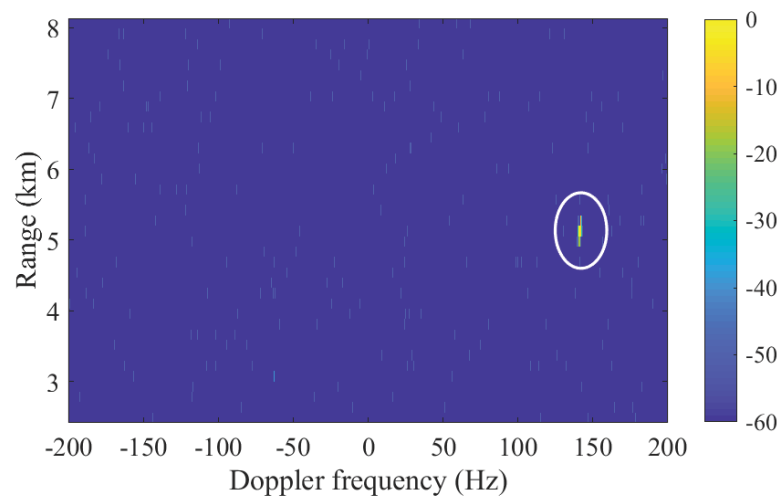

(b) Range-Doppler map obtained by averaging over 100 trials Fig. 7. Proposed algorithm combining the batches algorithm with compressive sensing run on $2^{11}$ randomly selected batches: (a) the ISL against different number of trials and (b) range-Doppler map obtained by averaging over 100 random selections (the white circle indicates target).

In addition, the computational complexity of the proposed algorithm with different numbers of selected batches is given in Fig. 8 (a). We can see that the computation time of the proposed algorithm with $2^{11}$ batches is smaller than the integration time, providing a real-time display. Fig. 8 (b) shows the ISL of the range-Doppler maps obtained by the proposed algorithm with different numbers of batches obtained by 100 random selections and average processing. As can be seen from Fig. 8 (b), the sidelobe level of the proposed algorithm with $2^{11}$ batches is smaller than the batches algorithm with $2^{14}$ batches. Besides, by offsetting the burden of CS by considering 8 times $\left(2^{11}\right.$ vs $\left.2^{14}\right)$ fewer cross-correlations for range compression, the proposed algorithm also have comparable processing time with the batches algorithm, as shown in Fig. 8 (a).

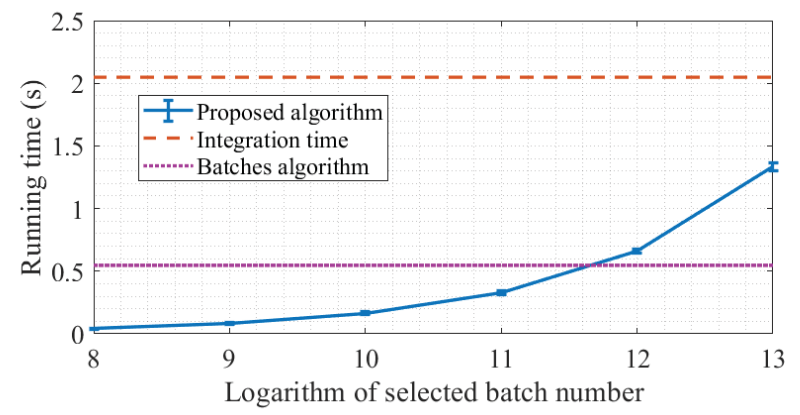

(a) Computation running time

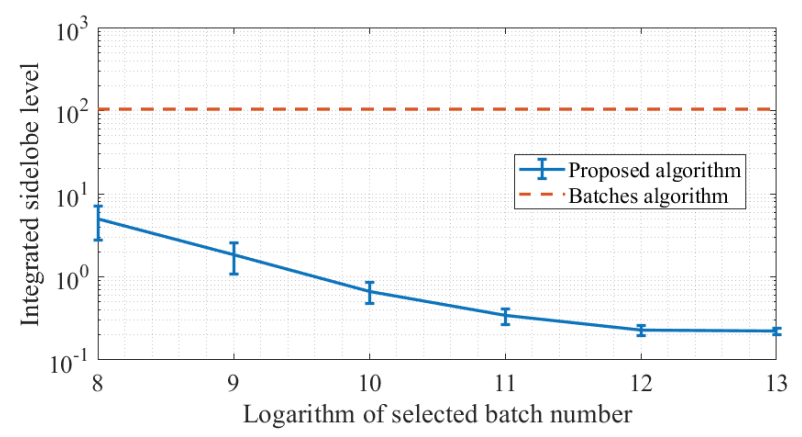

(b) Sidelobe level

Fig. 8. (a) Computation running time as a function of selected batch number, demonstrating real-time capability for all cases. (b) Sidelobe level of the proposed algorithm as a function of selected batch number and comparison with the sidelobe level of classical batches algorithm with $2^{14}$ batches.

For a more illustrative comparison of the proposed algorithm with the batches algorithm, two cuts are made along the location of the airplane in the Doppler and range directions shown in Fig. 4 (b) and Fig. 7 (b), and the results are shown in Fig. 9. The proposed algorithm has a considerably lower sidelobe level than the batches algorithm, confirming the former's superiority.

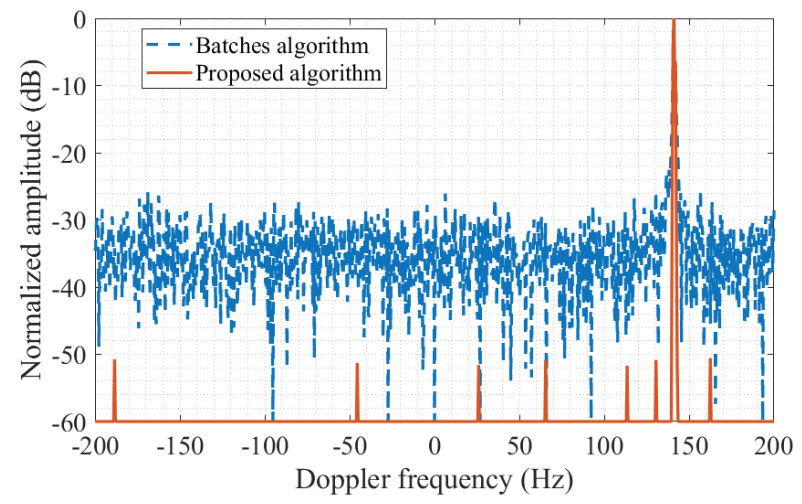

(a) Doppler direction 


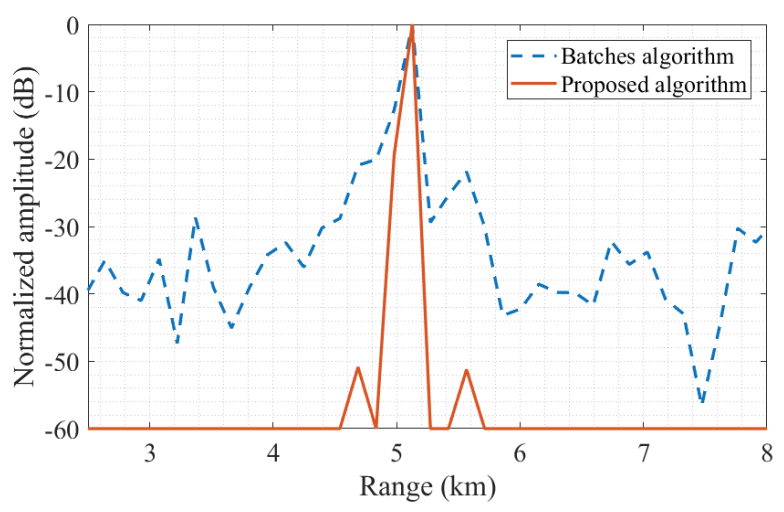

(b) Range direction

Fig. 9. Sidelobe level comparison between the batches algorithm and the proposed algorithm in (a) Doppler direction and (b) range direction.

Finally, about $1 \mathrm{~min}$ of the data measured to track the airplane is processed using the proposed algorithm, and the result is shown in Fig. 10. The white rectangle indicates the flying path of the airplane. It can be seen that the landing route of the airplane can be effectively estimated by the proposed algorithm within a $20 \mathrm{~dB}$ dynamic range.

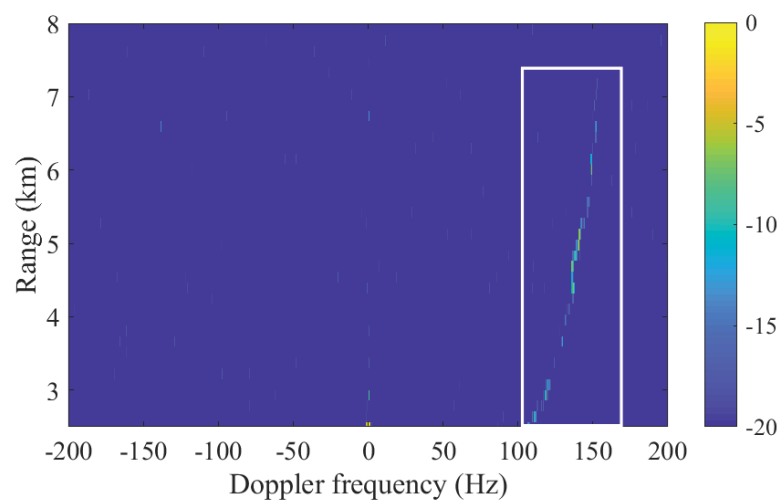

Fig. 10. Airplane tracking in the Doppler-range plane using 1-min data processed by the proposed algorithm.

\section{B. Large cross-section and slow moving targets: ships}

The second demonstration aims at detecting ships entering and leaving the port of Sendai. The objective is to demonstrate the multiple target tracking capability and target separation capacity of the proposed algorithm. Ships are selected because of their lower speed, leading to small Doppler frequencies that are closer to the stationary clutter.

The same analysis sequence as that used above for the airplane measurements is employed for the case of two large ships crossing each other, creating both positive and negative Doppler shifts. Fig. 11 (a) shows the range-Doppler map obtained from the classical batches algorithm. Fig. 11 (b) exhibits the same map obtained using the proposed algorithm, and its result is consistent with that shown in Fig. 11 (a), but the targets are better resolved. Again, background, calculated as the mean value of all batches, is subtracted from each batch, and the Doppler estimation results are obtained from the Fourier transform along the slow time direction.

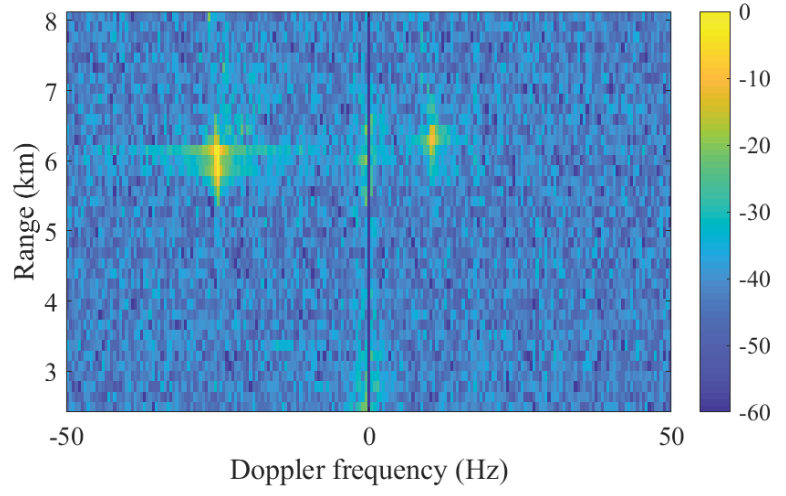

(a) Classical batches algorithm

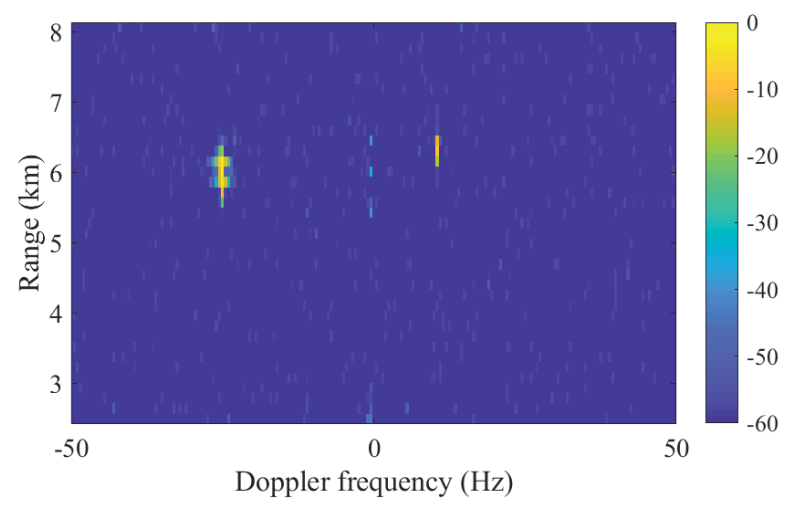

(b) The proposed algorithm

Fig. 11. Range-Doppler maps obtained by the (a) classical batches algorithm and (b) the proposed algorithm: both ships are visible in a range of $6 \mathrm{~km}$, one exhibiting a radial velocity away from the receiver (stronger target) and the other toward the receiver (weaker target).

Fig. 12 demonstrates the sidelobe level reduction achieved by the proposed algorithm as a cross-section along the abscissa of Figs. 11 (a) and Fig. 11 (b) for a range in which the two ships cross. Targets moving at different speeds are better separated by the proposed algorithm than by the classical batches algorithm, as shown by the comparison of the two methods in Fig. 13 (a) and Fig. 13 (b). To emphasize this result, a short integration time of $64 \mathrm{~ms}$ (as opposed to $2.048 \mathrm{~s}$ selected previously) was used, decreasing the velocity resolution to a level at which the two targets are mixed when the batches algorithm is used. The $64 \mathrm{~ms}$ duration is selected to mix the targets in the Doppler direction, while the practical application would aim at demonstrating separation of targets moving at slightly different speeds, inducing Doppler frequency differences below the inverse of the integration time.

Indeed, despite some suggestions of two maxima in Fig. 14 (blue-dashed curve), the right-most maximum obtained by the classical batches algorithm is not located at the correct speed but at $20 \mathrm{~Hz}$, as opposed to that obtained by the proposed algorithm, which properly identifies the weaker target velocity at $10 \mathrm{~Hz}$, as is shown with the longer integration time in Fig. 12. The extended Doppler frequency range of Fig. 14 is selected to illustrate that the sidelobe peak is stronger than the weaker target when using the classical batches algorithm, as indicated by the black circle in Fig. 14. 
The range-Doppler tracking of the two ships during a 2.7min-long acquisition is shown in Fig. 15, where the routes of two ships can be clearly observed. Thus, the new algorithm indeed allows for improved separation of multiple targets characterized by different Doppler shifts.

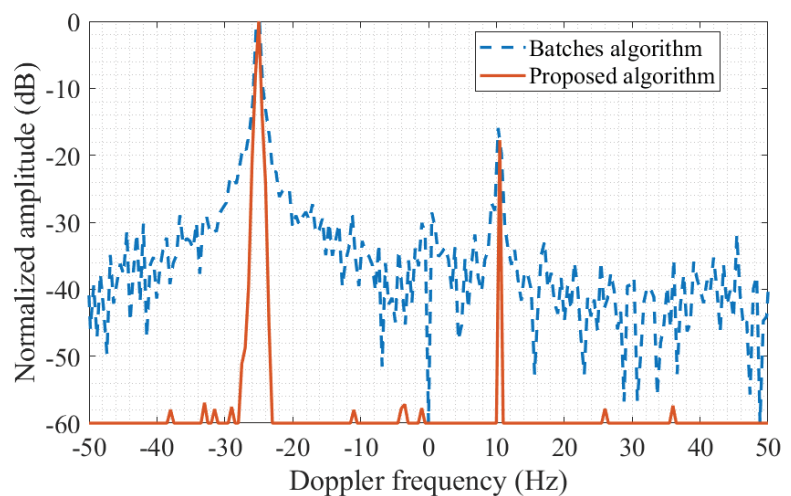

Fig. 12. Sidelobe level reduction as observed from cross-sections of the rangeDoppler maps for a range in which both ships cross.

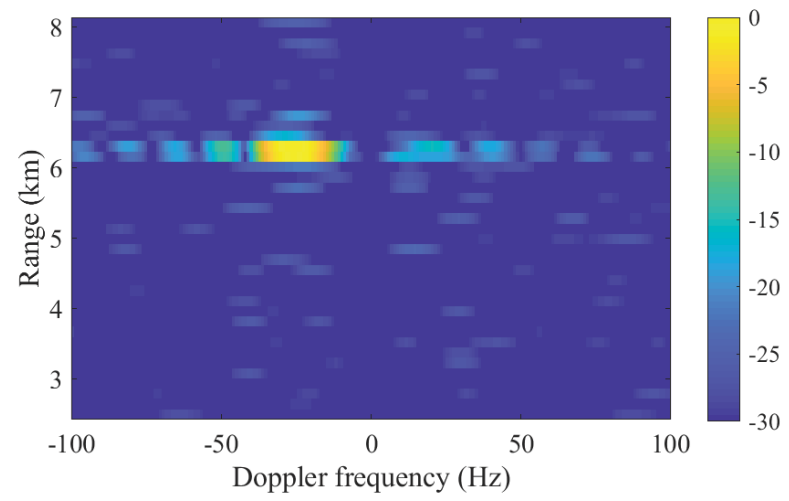

(a) Classical batches algorithm

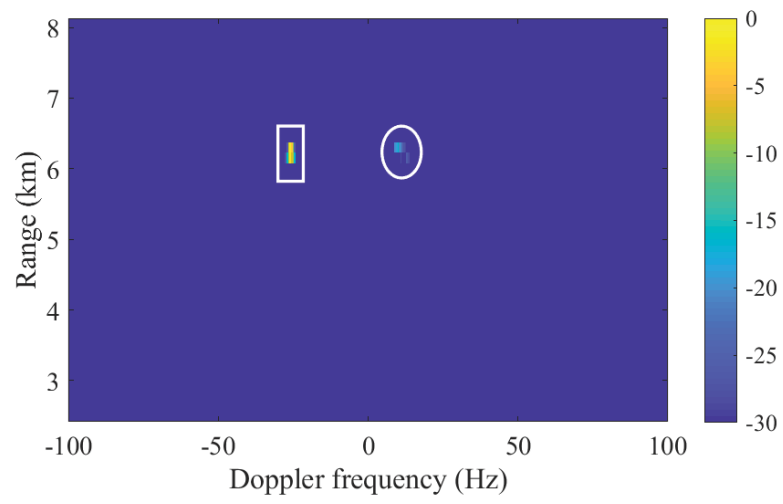

(b) The proposed algorithm

Fig. 13. Range-Doppler map resulting from the analysis of a short integration time subset using (a) the classical batches algorithm, demonstrating the degraded Doppler resolution with mixed targets as the two ships cross, and (b) the proposed algorithm, demonstrating the ability to separate both targets despite the degraded Doppler resolution due to the short integration time.

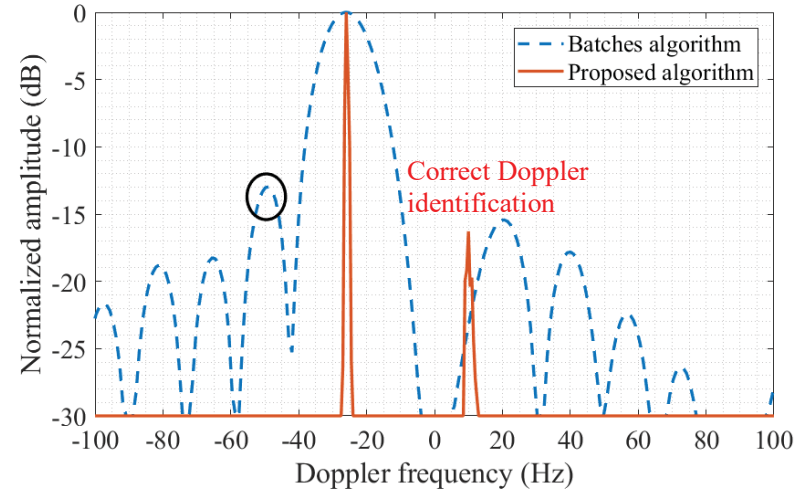

Fig. 14. Quantitative analysis of the Doppler resolution improvement and ability to separate targets using the proposed algorithm.

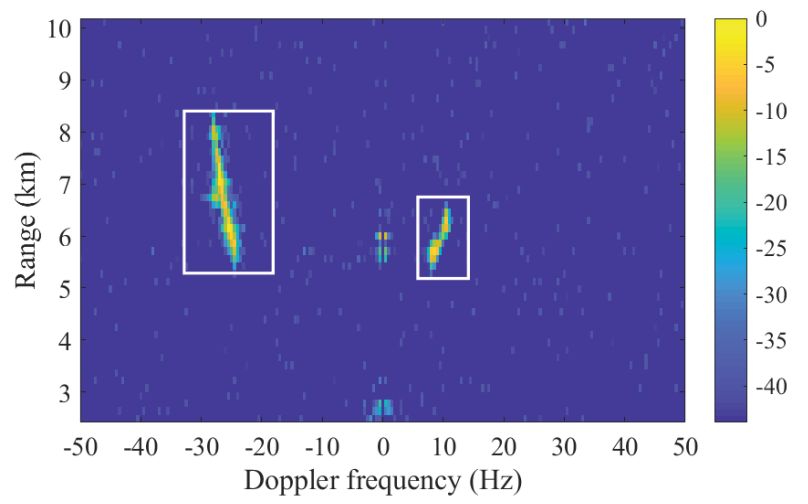

Fig. 15. Tracking of two ships for a duration of 2.7 min: the left ship moves away from the receiver and the right ship moves toward the receiver.

\section{CONCLUSION}

We have proposed a novel real-time range-Doppler map generation algorithm for PBR applications. We investigated low-cost PBR consisting of synchronized DVB-T receivers used as general-purpose software-defined radio sources. A low sampling rate of $2.048 \mathrm{Msamples} / \mathrm{s}$ is used to demonstrate the performance of the proposed batch CS algorithm for detecting and tracking moving targets. Following the detailed description of the theoretical background and the novel developments, the algorithm was employed using moving airplanes and ships as targets. The proposed algorithm showed an improved range and Doppler sidelobe level reduction compared to the classical batches algorithm while allowing real-time processing, as the computation duration was shorter than the acquisition duration. The improved Doppler resolution helped to improve the target separation capability, as demonstrated in the example of two ships crossing each other. The designed low-cost PBR system and the proposed algorithm have potentials to be applied in airplane navigation and harbor protection. The further study will focus on the extension of the proposed algorithm for the simultaneous estimation of target range, velocity and azimuth information, which will increase the capacity of the PBR system. In addition, CS theory will be employed for range resolution improvement for short-range moving target detection and tracking, which is beneficial for some applications, such as traffic density monitoring. 


\section{ACKNOWLEDGMENT}

This work is supported by JSPS Grant-in-Aid for Scientific Research (A) 26249058. J.-MF is grateful to Tohoku University for granting a position as a three-month visiting scientist to the Center for Northeast Asian Studies. The DVB-T passive radar investigation is partly supported by the French Centre National de la Recherche Scientifique (CNRS) PEPS grant.

\section{REFERENCES}

[1] J. Palmer, S. Palumbo, A. Summers, D. Merrett, S. Searle, and S. Howard, "An overview of an illuminator of opportunity passive radar research project and its signal processing research directions," Digital Signal Processing, vol. 21, no. 5, pp. 593-599, 2011.

[2] P. E. Howland, H. D. Griffiths, and C. J. Baker, "Passive bistatic radar systems," Bistatic Radar: Emerging Technology, 2008.

[3] P. E. Howland, D. Maksimiuk, and G. Reitsma, "Fm radio based bistatic radar," IEE Proceedings-Radar, Sonar and Navigation, vol. 152, no. 3, pp. 107115, 2005.

[4] C. Coleman and H. Yardley, "Passive bistatic radar based on target illuminations by digital audio broadcasting," IET Radar, Sonar \& Navigation, vol. 2, no. 5, pp. 366-375, 2008.

[5] H. Ma, M. Antoniou, D. Pastina, F. Santi, F. Pieralice, M. Bucciarelli, and M. Cherniakov, "Maritime moving target indication using passive GNSS-based bistatic radar," IEEE Transactions on Aerospace and Electronic Systems, vol. 54, no. 1, pp.115-130, 2018.

[6] Z. Li, F. Santi, D. Pastina, and P. Lombardo, "Passive radar array with lowpower satellite illuminators based on fractional Fourier transform," IEEE Sensors Journal, vol. 17, no. 24, pp. 8378-8394, 2017.

[7] F. Colone, P. Falcone, and C. Bongioanni, "WiFi-based passive bistatic radar: Data processing schemes and experimental results," IEEE Transactions on Aerospace and Electronic Systems, vol. 48, no. 2, pp. 1061-1079, 2012.

[8] W. Feng, J. M. Friedt, G. Goavec-Merou, and M. Sato, "Passive radar delay and angle of arrival measurements of multiple acoustic delay lines used as passive sensors," IEEE Sensors Journal, vol. 19, no. 2, pp. 594-602, 2018.

[9] D. Gromek, K. Kulpa, and P. Samczynski, "Experimental results of passive SAR imaging using DVB-T illuminators of opportunity," IEEE Geoscience and Remote Sensing Letters, vol. 13, no. 8, pp. 1124-1128, 2016.

[10] J. E. Palmer, H. A. Harms, S. J. Searle, and L. Davis, "DVB-T passive radar signal processing," IEEE transactions on Signal Processing, vol. 61, no. 8, pp. 2116-2126, 2013.

[11] B. Dawidowicz, P. Samczynski, M. Malanowski, J. Misiurewicz, and K. Kulpa, "Detection of moving targets with multichannel airborne passive radar," IEEE Aerospace and Electronic Systems Magazine, vol. 27, no. 11, pp. 42-49, 2012.

[12] P. Wojaczek, F. Colone, D. Cristallini, and P. Lombardo, "Reciprocal filter-based STAP for passive radar on moving platforms," IEEE Transactions on Aerospace and Electronic Systems, 2018.

[13] D. Gromek, K. Radecki, J. Drozdowicz, P. Samczyński, and J. Szabatin, "Passive SAR imaging using DVB-T illumination for airborne applications," IET Radar, Sonar \& Navigation, 2018.

[14] M. Martorella and E. Giusti, "Theoretical foundation of passive bistatic ISAR imaging," IEEE Transactions on Aerospace and Electronic Systems, vol. 50, no. 3, pp. 1647-1659, 2014.

[15] D. Olivadese, E. Giusti, D. Petri, M. Martorella, A. Capria, and F. Berizzi, "Passive ISAR with DVB-T signals," IEEE Transactions on Geoscience and Remote Sensing, vol. 51, no. 8, pp. 4508-4517, 2013.

[16] S. Stein, "Algorithms for ambiguity function processing," IEEE Transactions on Acoustics, Speech, and Signal Processing, vol. 29, no. 3, pp. 588-599, 1981.

[17] D. Langellotti, F. Colone, C. Bongioanni, and P. Lombardo, "Comparative study of ambiguity function evaluation algorithms for passive radar," in International Radar Symposium IRS 2009, 2009, pp. 325-329.
[18] C. Moscardini, D. Petri, A. Capria, M. Conti, M. Martorella, and F. Berizzi, "Batches algorithm for passive radar: A theoretical analysis," IEEE Transactions on Aerospace and Electronic Systems, vol. 51, no. 2, pp. 14751487, 2015.

[19] R. G. Baraniuk, “Compressed sensing,” IEEE signal processing magazine, vol. 24, no. 4, pp. 118-121, 2007.

[20] J. H. Ender, "On compressed sensing applied to radar," Signal Processing, vol. 90, no. 5, pp. 1402-1414, 2010.

[21] M. Weiss, "Sparsity-based radar technique," Academic Press Library in Signal Processing, vol. 7, pp. 277-316, 2018.

[22] H. Biao, G. Zhang, Z. Li, and L. Jiao, "Sparse coding-inspired highresolution ISAR imaging using multistage compressive sensing," IEEE Transactions on Aerospace and Electronic Systems, vol. 53, no. 1, pp. 26-40. 2017.

[23] W. Qiu, E. Giusti, A. Bacci, M. Martorella, F. Berizzi, H. Zhao, and Q. Fu, "Compressed sensing-based algorithm for passive bistatic ISAR with DVB-T signals," IEEE Transactions on Aerospace and Electronic Systems, vol. 51, no. 3, pp. 2166-2180, 2015.

[24] M. S. Kang, S. H. Lee, K. T. Kim, and J. H. Bae, "Bistatic ISAR imaging and scaling of highly maneuvering target with complex motion via compressive sensing," IEEE Transactions on Aerospace and Electronic Systems, vol. 54, no. 6, pp. 2809-2826, 2018.

[25] X. Dong and Y. Zhang, "A novel compressive sensing algorithm for SAR imaging," IEEE Journal of Selected Topics in Applied Earth Observations and Remote Sensing, vol. 7, no. 2, pp. 708-720, 2013.

[26] Q. Wu, Y. D. Zhang, M. G. Amin, and B. Himed, "High-resolution passive SAR imaging exploiting structured Bayesian compressive sensing," IEEE Journal of Selected Topics in Signal Processing, vol. 9, no. 8, pp. 1484-1497, 2015.

[27] W. Feng, L. Yi, and M. Sato, "Near range radar imaging based on block sparsity and cross-correlation fusion algorithm," IEEE Journal of Selected Topics in Applied Earth Observations and Remote Sensing, vol. 11, no. 6, pp. 2079-2089, 2018.

[28] K. Duan, Z. Wang, and W. Xie, "Sparsity-based STAP algorithm with multiple measurement vectors via sparse Bayesian learning strategy for airborne radar," IET Signal Processing, vol. 11, no. 5, pp. 544-553, 2017.

[29] Z. Yang, RC. de Lamare, and W. Liu, "Sparsity-based STAP using alternating direction method with gain/phase errors," IEEE Transactions on Aerospace and Electronic Systems, vol. 53, no. 6, pp. 2756-2768, 2017.

[30] L. Zheng and X. Wang, "Super-resolution delay-Doppler estimation for OFDM passive radar," IEEE Transactions on Signal Processing, vol. 65, no. 9, pp. 2197-2210, 2017.

[31] R. A. Sevimli, M. Tofighi, A. E. Cetin, "Range-Doppler radar target detection using denoising within the compressive sensing framework," in Signal Processing Conference (EUSIPCO), 2014 Proceedings of the 22nd European, IEEE, pp. 1950-1954, 2014.

[32] M. Weiß, "Compressed sensing for passive surveillance radar using dab signals," in IEEE International Radar Conference, pp. 1-6, 2014.

[33] M. A. Hadi, M. N. Tabassum, and S. Alshebeili, "Compressed sensing based high-resolution passive bistatic radar," Signal, Image and Video Processing, vol. 11, no. 4, pp. 635-642, 2017.

[34] W. Feng, J.-M. Friedt, G. Cherniak, and M. Sato, "Passive bistatic radar using digital video broadcasting-terrestrial receivers as general-purpose software-defined radio receivers," Review of Scientific Instruments, vol. 89, no. 10, 104701, 2018.

[35] J. A. Tropp and A. C. Gilbert, "Signal recovery from random measurements via orthogonal matching pursuit," IEEE Transactions on information theory, vol. 53, no. 12, pp. 4655-4666, 2007.

[36] H. Mohimani, M. Babaie-Zadeh, and C. Jutten, "A fast approach for overcomplete sparse decomposition based on smoothed 10 norm," IEEE Transactions on Signal Processing, vol. 57, no. 1, pp. 289-301, 2009.

[37] S. S. Chen, D. L. Donoho, and M. A. Saunders, "Atomic decomposition by basis pursuit,” SIAM review, vol. 43, no. 1, pp. 129-159, 2001. 
[38] Y. Ma, T. Shan, Y. D. Zhang, M. G. Amin, R. Tao, and Y. Feng, "A novel two-dimensional sparse-weight nlms filtering scheme for passive bistatic radar," IEEE Geoscience and Remote Sensing Letters, vol. 13, no. 5, pp. 676-680, 2016.

[39] J. L. Garry, C. J. Baker, and G. E. Smith, "Evaluation of direct signal suppression for passive radar," IEEE Transactions on Geoscience and Remote Sensing, vol. 55, no. 7, pp. 3786-3799, 2017.

[40] D. Needell and J. A. Tropp, "Cosamp: Iterative signal recovery from incomplete and inaccurate samples," Applied and Computational Harmonic Analysis, vol. 26, no. 3, pp. 301-321, 2009.

[41] D. L. Donoho, Y. Tsaig, I. Drori, and J.-L. Starck, "Sparse solution of underdetermined systems of linear equations by stagewise orthogonal matching pursuit," IEEE Transactions on Information Theory, vol. 58, no. 2, pp. 10941121, 2012.

[42] S. Ji, Y. Xue, and L. Carin, "Bayesian compressed sensing," IEEE Transactions on Signal Processing, vol. 56, no. 6, pp. 2346-2356, 2008.

[43] E. J. Candes, J. Romberg, and T. Tao, "Robust uncertainty principles: Exact signal reconstruction from highly incomplete frequency information," IEEE Transactions on information theory, vol. 52, no. 2, pp. 489-509, 2006.

[44] T. W. Rondeau, "On the GNU Radio ecosystem," Opportunistic Spectrum Sharing and White Space Access: The Practical Reality, pp. 25-48, 2015. 